\title{
A Detailed Morphological and Spectroscopic Study of Merging Dwarf Galaxy PGC 030133
}

\author{
D. N. Chhatkuli, S. Paudel and B. Aryal
}

\section{Journal of Nepal Physical Society}

Volume 7, Issue 4, December 2021

ISSN: 2392-473X (Print), 2738-9537 (Online)

\section{Editors:}

Dr. Binod Adhikari

Dr. Bhawani Joshi

Dr. Manoj Kumar Yadav

Dr. Krishna Rai

Dr. Rajendra Prasad Adhikari

Mr. Kiran Pudasainee

JNPS, 7 (4), 28-35 (2021)

DOI: http://doi.org/10.3126/jnphyssoc.v7i4.42928

$$
\text { JNPS }
$$

ISSN:2392-473X (Print) 2738-9537 (Online)

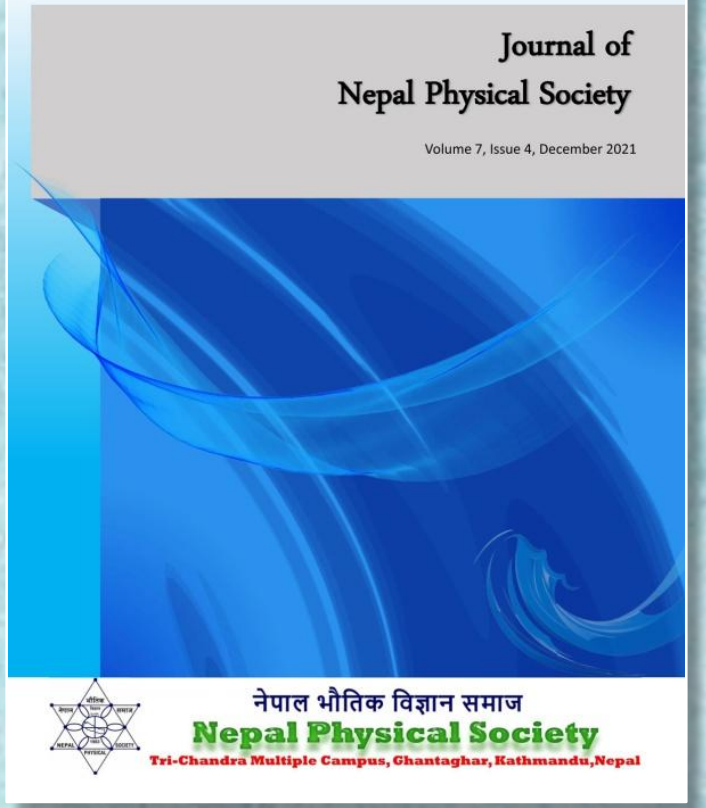

Published by:

Nepal Physical Society

P.O. Box: 2934

Tri-Chandra Campus

Kathmandu, Nepal

Email:nps.editor@gmail.com 


\title{
A Detailed Morphological and Spectroscopic Study of Merging Dwarf Galaxy PGC 030133
}

\author{
D. N. Chhatkuli ${ }^{1, *}$, S. Paudel ${ }^{2}$ and B. Aryal ${ }^{1}$ \\ ${ }^{1}$ Central Department of Physics, Tribhuvan University, Nepal \\ ${ }^{2}$ Centre for Galaxy Evolution Research, Department of Astronomy, Yonsei University, \\ Seoul, South Korea \\ *Corresponding Email: chhatkulidn@gmail.com
}

Received: 20th November, 2021; Revised: 19th December, 2021; Accepted: 29th December, 2021

\begin{abstract}
We present a detailed morphology and spectroscopic study of a merging dwarf galaxy PGC 030133. Using the publicly available fiber spectroscopic data from the Sloan Digital Sky Survey (SDSS), we analyzed nine-strong emission lines of wavelength range $4336 \AA$ to $6739 \AA$. We find that the strongest emission line is $\mathrm{OIII}_{5007}$, with an intensity of $146.32 \times 10^{-17} \mathrm{erg} / \mathrm{s} / \mathrm{cm}^{2} / \AA$. The observed emission lines are well fitted with a Gaussian profile with a coefficient of regression greater than $96 \%$, and the derived full-width half maximum (FWHM) is less than $4.2 \AA$. The Balmer decrement, characterized by the line ratio between $\mathrm{H}_{\alpha}$ and $\mathrm{H}_{\beta}$ is 3.07 , suggesting a presence of dust at the center of PGC 030133. Using extinction corrected emission line fluxes, we derived the star-formation rate and emission line metallicity of PGC 030133. SFR derived from $\mathrm{H}_{\alpha}$ emission line flux is $0.0033 \mathrm{M}_{\odot}$ year $^{-1}$ and emission line metallicity derived from flux ratio between NII and $\mathrm{H}_{\alpha}$ is 8.13 dex. We derived morphological parameters using the SDSS, g, and zband imaging data. PGC 030133 has a half-light radius of 3.38 arc second and 3.05 arc second in g-band and z-band respectively and observed one-dimensional light profile is well fitted with a Sersic function with near exponential Sersic index $\sim 0.9$.
\end{abstract}

Keywords: Compact dwarf galaxy, Galaxy merger, Half-light radius, $\mathrm{H}_{\alpha}$ line, Star Formation Rate.

\section{INTRODUCTION}

In a galaxy merger, the interacting galaxies combine into a single galaxy that contains stars, planets, dust, and gas. In the gas clouds, formed after the merger, stars will be formed at higher rates. From the observational studies in the last ten years, it has been established that the rate of the merger of galaxies increases steadily with redshift up to $\mathrm{z} \sim 2-3[1]$. Galaxy merger in the local universe is not common to those in the early universe. Therefore, the galaxy merger in the local universe gives an in-depth examination of the role of the merger in the evolution of the galaxy. Due to the limited existing surveys of the merger between dwarf galaxies $\left(\mathrm{M}_{*}<10^{9} \mathrm{M}_{\odot}\right)$, most of the study of galaxy mergers so far has been concentrated on massive galaxies. It is because, the difficulty in examining the dwarf galaxies is partly due to their low luminosity, low brightness, and swallow potential. The galaxy merger enhances the morphological transformations as well as the star formation and enhances far infra-red emission [2]. The extreme enhancement of star formation can produce a starburst $\sim 1000 \mathrm{M}_{\odot} \mathrm{yr}^{-1}[3,4]$. In the starburst regime, the total gas budget is dominated by $\mathrm{H}_{2}$ gas. Star formation is one of the most important processes in the formation and evolution of the galaxy. In low redshift galaxies, star formation in spiral galaxies occurs mainly in molecular clouds. Starbursts occur in a special class of galaxies called blue compact dwarf galaxies (BCDs). Starburst galaxies are gas-rich and use their gas much faster to form stars. It is anticipated that the gas component in interacting systems is frequently predicted to be more turbulent than an isolated system.

A compact group of galaxies is a dense group of galaxies that undergo into merger after the 
interaction. Zwicky [5] reported that the collision of the stars increases frequently when the compactness of a galaxy increases and the matter is ejected in different forms. When two galaxies with compact nuclei collide, an isolated compact galaxy will be formed [6]. Observationally, it is found that the compact galaxies are related to normal galaxies, compact galaxies, or even with both of them. Some galaxies are highly compact, called Ultra Compact Dwarf galaxies (UCDs) [7], the sizes, velocity dispersion, and luminosities of which are similar to the dwarf galaxy cores but they have a higher massto-light ratio than even the largest globular clusters [8]. The blue compact dwarf galaxies (BCDs) are the blue colored galaxies having enormous starburst activities and normally higher central surface brightness in comparison to ordinary dwarf irregular galaxies [9], which is explained to be formed by the gas-rich dwarf-dwarf mergers [10, 11]. The BCDs may also be formed by the central starburst supported by the natural gas accretion from the local environment $[12,13]$.

In this work, the spectroscopic and photometric analysis of a star-forming interacting dwarf galaxy PGC 030133 is performed. The star formation rate (SFR) and line metallicity are calculated by using
$\mathrm{H}_{\alpha}$ line flux in spectroscopy while the structural parameters like effective radius, half-light radius, and ellipticity are calculated in photometry.

\section{MATERIALS AND METHODS}

\subsection{Sample Selection}

A star-forming class of interacting dwarf galaxies, PGC 030133 is selected from the catalog prepared by Paudel et al. [14] based on low redshift ( $\mathrm{z}=$ 0.0036) and compactness. The galaxy PGC 030133 is located in the sky position R. A. (J2000 = 10:19:01.49) and Dec. (J2000 = +21:17:00.96) at an adopted distance of $24.80 \mathrm{Mpc}$ with a line-of-sight radial velocity of $1080 \mathrm{~km} / \mathrm{s}$. Its $\mathrm{g}$ and r-band magnitudes are 14.56 mag. and 14.34 mag. respectively, and the total absolute B-band magnitude is $-17.11 \mathrm{mag}$. The logarithmic value of the stellar mass of the galaxy is $8.86 \mathrm{M}_{\odot}$. The $g-r-i$ combined tri-color image of the galaxy PGC 030133 obtained from the Legacy survey is shown in Fig. 1(a). The galaxy has compact morphology with its bright center as compared to the outer part. A reddish, extended tail-like structure can be seen towards the north direction emanated from the galaxy.
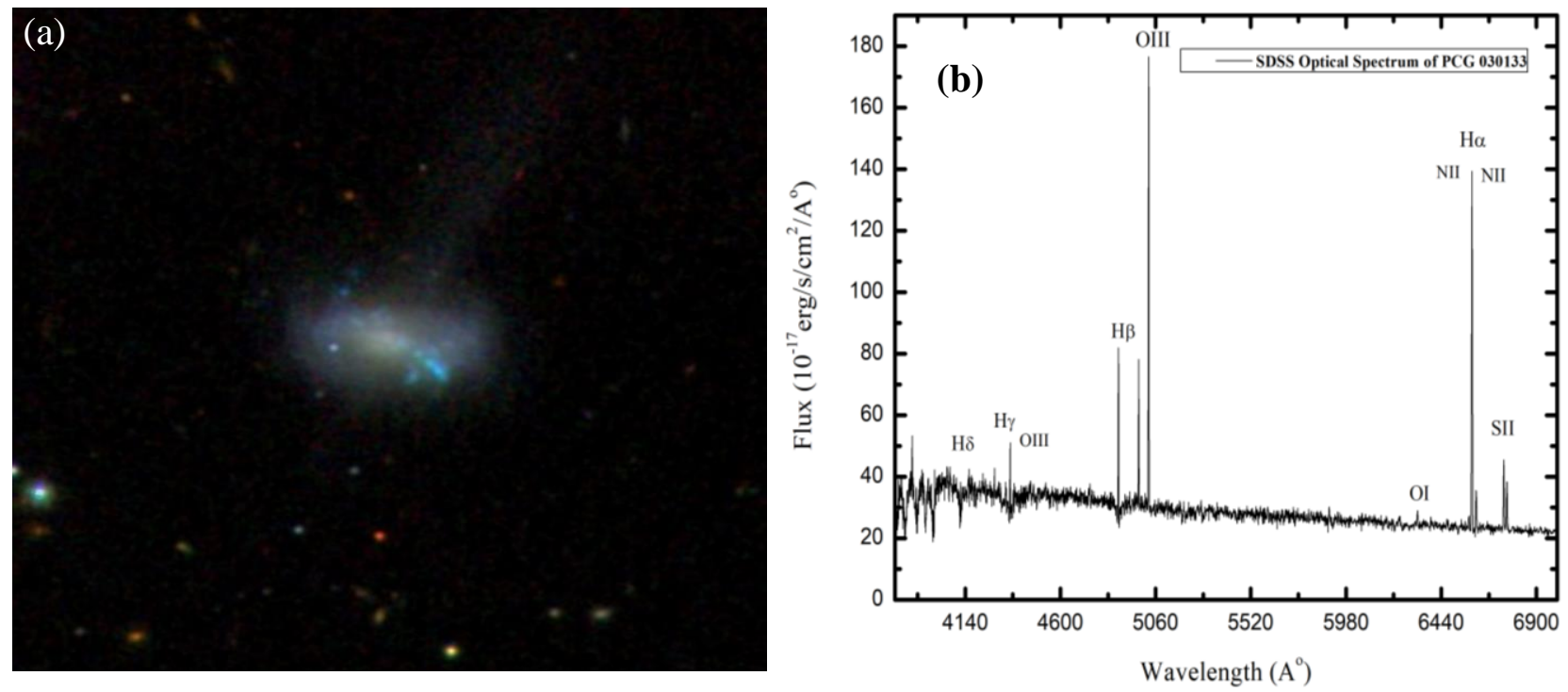

Fig. 1: (a) Optical image of PGC 030133. The image is obtained from the SDSS sky-server. (b) The optical spectrum of the galaxy PGC 030133. Position of the emission lines $\mathrm{H}_{\alpha}, \mathrm{H}_{\beta}, \mathrm{H}_{\gamma}, \mathrm{H}_{\delta}$, doublet OIII, OI, SII, and doublet NII are identified and are shown in the spectrum. The $\mathrm{X}$-axis is rest-frame wavelength and $\mathrm{Y}$-axis is flux.

In Fig. 1(b), the SDSS optical spectrum of the galaxy PGC 030133 obtained from the SDSS archival data is shown in the wavelength range of $3800 \AA$ to $7000 \AA$. Several prominent emission lines such as $\mathrm{H}_{\alpha}, \mathrm{H}_{\beta}, \mathrm{H}_{\gamma}, \mathrm{H}_{\delta}$, doublet OIII, OI, SII, and doublet NII in the spectrum can be seen along with some small absorption lines and highlighted some of the prominent emission lines, mainly Balmer lines. The strongest emission line is $\mathrm{OIII}_{5007}$, and the second strong line is $\mathrm{H}_{\alpha}$. 


\subsection{Data Analysis}

\section{(a) Spectroscopic Analysis}

In this work, we tend to largely use the Sloan Digital Sky Survey (SDSS) archival data to measure the morphological and chemical parameters of the galaxies. The SDSS Data Archive Server (DAS) is used to get the SDSS g and r-band images and fiber-spectrum which are already wellcalibrated. Both images and spectrum data are in fits file format. The SDSS fiber has a diameter of three arc seconds. Therefore, only a tiny fraction of the galaxy area of the central region is covered by the spectroscopic data. The spectral line profile is considered to be gaussian in nature and the Gaussian distribution function is defined by Squires et al. [15] as

$$
f_{G}(x)=\frac{1}{\sqrt{2 \pi \sigma^{2}}} e^{\frac{-(x-\mu) 2}{2 \sigma^{2}}}
$$

Where, $x$ is a normal random variable, $\mu$ is the mean deviation and $\sigma$ is the standard deviation of the distribution.

Some strongest emission lines will be presented in this paper, along with their Gaussian parameters enlisted in a separate table. Then the corresponding elements will be identified. A median of the flux of the spectrum range \pm 100 from the center of the emission line was taken and subtracted the continuum flux before fitting each of the emission lines. Furthermore, the star formation rate (SFR), the hydrogen line ratio, metallicity, dust extinction will be calculated. The Gaussian area of the $\mathrm{H}_{\alpha}$ emission line will be used to calculate the star formation rate of the galaxy. The SFR will be calculated by adopting the empirical formula proposed by Kennicutt [16] as follows:

$$
\operatorname{SFR}\left(\mathrm{M}_{\odot} \text { year }^{-1}\right)=7.9 \times 10^{-42} \sum \mathrm{L}\left(\mathrm{H}_{\alpha}\right)\left(\operatorname{ergs~s}^{-1}\right)
$$

Where $\sum \mathrm{L}\left(\mathrm{H}_{\alpha}\right)$ is the total luminosity of the $\mathrm{H}_{\alpha}$ line which will be calculated by using the area of the Gaussian fits. The calibration provided by Marino et al. [17] will be used to calculate the emission line metallicity by using a line ratio between $\mathrm{H}_{\alpha}$ and NII which is given by

$$
12+\log (\mathrm{O} / \mathrm{H})=8.743+0.462 \times \log \left(\mathrm{NII} / \mathrm{H}_{\alpha}\right)
$$

The line ratio between $\mathrm{H}_{\alpha}$ and $\mathrm{H}_{\beta}$ fluxes is called Blamer decrement $\mathrm{c}$. The theoretical value of $\mathrm{c}_{0}=$ 2.86 for an electron temperature of $10^{4} \mathrm{~K}$ and electron density of $10^{2} \mathrm{~cm}^{-3}[18,19]$. Now extinction coefficient $\mathrm{E}(\mathrm{B}-\mathrm{V})=1.97 \times \log \left(\mathrm{c} / \mathrm{c}_{0}\right)$.
Total extinction $\mathrm{A}\left(\mathrm{H}_{\alpha}\right)=2.45 \mathrm{E}(\mathrm{B}-\mathrm{V})$ in $\mathrm{H}_{\alpha}$ band. Also, the emitted and observed fluxes of $\mathrm{H}_{\alpha}$ can be related as $\mathrm{A}\left(\mathrm{H}_{\alpha}\right)=-2.5 \log \left[\mathrm{F}\left(\mathrm{H}_{\alpha}\right)_{\text {obs }} / \mathrm{F}\left(\mathrm{H}_{\alpha}\right)_{\mathrm{em}}\right]$. Then the emitted flux, $\mathrm{F}\left(\mathrm{H}_{\alpha}\right)_{\mathrm{em}}$ can be calculated which will be used to calculate the SFR after extinction correction.

\section{(b) Photometric Analysis}

For photometric analysis, we do surface photometry on the r-band optical image of the galaxy because of its higher signal-to-noise ratio as compared to other bands. The major axis light profile of the galaxy is extracted by using the Image Reduction and Analysis Facility (IRAF) task ellipse and the best fitted elliptical isophotes are drawn on the image as described by Jedrzejewski [20], thereby producing several structural parameters of the galaxy such as ellipticity, position angle, effective radius, intensity and so on which are important tools to know the morphology and evolution of the galaxy.

To find the size of a galaxy, we use either a parametric approach or a non-parametric approach. In the former approach, the observed light profile of the galaxy is approximated into parametric functions such as exponential or de Vaucouleur functions which are the special cases of Sersic function [21] defined as

$$
\mathrm{I}(\mathrm{R})=\mathrm{I}_{e} \exp \left\{-\mathrm{b}_{\mathrm{n}}\left[\left(\frac{\mathrm{R}}{\mathrm{R}_{e}}\right)^{1 / \mathrm{n}}-1\right]\right\}
$$

Here, $\mathrm{R}_{e}$ is the effective radius of the galaxy at which the intensity is $\mathrm{I}_{e}$. $\mathrm{n}$ is called Sersic index which defines the 'shape' of the profile. $b_{n}$ is a function of $\mathrm{n}$ which is selected to make sure that the radius $\mathbf{R}_{e}$ encloses half of the profile's total luminosity. The observed light profile is modeled with the Sersic function and the best fitted effective radius $\mathrm{R}_{e}$ and surface brightness $\mu_{e}$ of the galaxy are obtained as free parameters.

Out of many methods in the non-parametric approach, the Petrosian method is used to find the half-light radius $\mathrm{R}_{h}$. At first, Petrosian radius $\mathrm{a}_{p},[22,23]$ is defined, which is the distance from the center of the galaxy at which the surface brightness at a radial distance $\mathrm{R}$ is 20 percent of the average surface brightness within that radius. It is assumed that the total flux of the galaxy is covered within the distance of $2 \mathrm{a}_{p}$. Once we know the total flux of the galaxy, the galactocentric distance at which total flux is half can be calculated, which is called half-light radius $\mathrm{h} r$. 


\section{RESULTS AND DISCUSSION}

\subsection{Spectroscopy}

Nine main emission lines: $\mathrm{H}_{\delta}, \mathrm{H}_{\beta}, \mathrm{OIII}_{4960}$, $\mathrm{OIII}_{5007}, \mathrm{H}_{\alpha}, \mathrm{NII}_{6584}, \mathrm{NII}_{6551}, \mathrm{SII}_{6717}$, and $\mathrm{SII}_{6733}$ are determined from the spectrum of the galaxy PGC 030133 [Fig. 1(b)] and only six prominent lines of them are shown in Fig. 2 along with their Gaussian fits. The solid line represents the Gaussian distribution and the dots represent the observed data. The error bars are also shown in the Gaussian curves. We can see that there is an excellent agreement of the observed data with the normal distribution. The doublets OIII, NII, and SII have two different emission lines of each. The Balmer lines, $\mathrm{H}_{\alpha}, \mathrm{H}_{\beta}$, and $\mathrm{H}_{\delta}$ are sensitive to the galaxies' gas-mass and star-formation rate. The $\mathrm{H}_{\alpha}$, principally, is a well-known tracer of star-formation and is widely used to calculate the star-formation rate of the gas-rich galaxies. Heavier elements OIII and NII emission fluxes are used to calculate the star-forming galaxies' emission line metallicity.
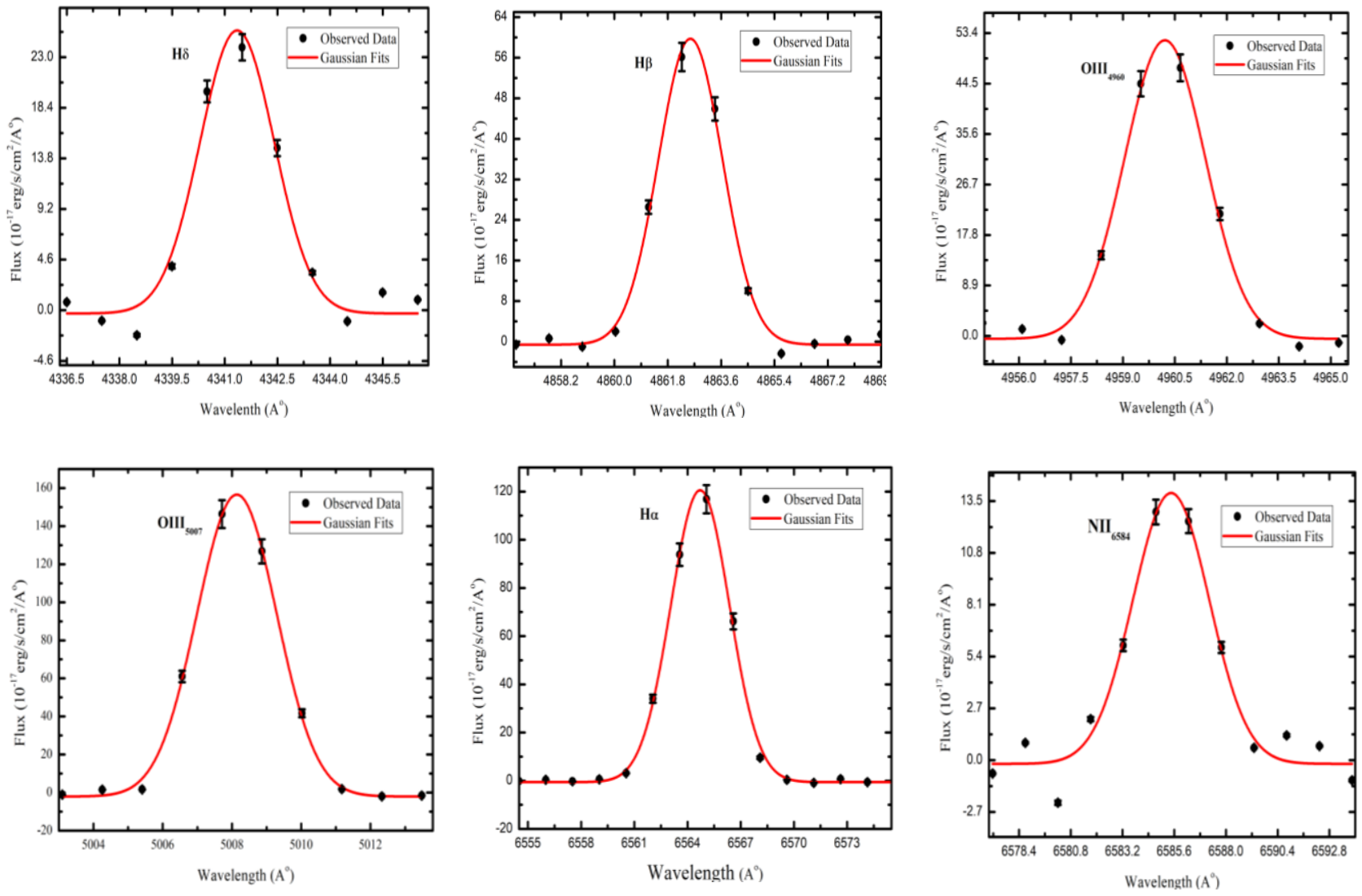

Fig. 2: Six main emission lines $\left(\mathrm{H}_{\delta}, \mathrm{H}_{\beta}, \mathrm{OIII}_{4960}, \mathrm{OIII}_{5007}, \mathrm{H}_{\alpha}\right.$ and $\left.\mathrm{NII}_{6584}\right)$ of Gaussian fitting procedure for the galaxy PGC 030133 are shown. We show a conservative estimate of the flux error in the plot, i.e., $10 \%$ of the observed flux provided by the SDSS webpage (https://www.sdss.org/dr15/spectro/caveats/). The major cause of the broadening of the characteristic line is the Doppler broadening. The wavelengths given in the $\mathrm{X}$-axis are redshift corrected.

The Gaussian parameters of the nine strongest emission lines of the galaxy PGC 030133 are enlisted in Table 1. The wavelength $\left(\lambda_{p}\right)$ in angstrom corresponding to the peak intensity, the value of peak intensity $\left(10^{-17} \mathrm{erg} / \mathrm{s} / \mathrm{cm}^{2} / \AA\right)$, area of the Gaussian curve $\left(10^{-17} \mathrm{erg} / \mathrm{s} / \mathrm{cm}^{2} / \AA\right)$, the height of the Gaussian fits $\left(10^{-17} \mathrm{erg} / \mathrm{s} / \mathrm{cm}^{2} / \AA\right)$, the positional difference between Gaussian peak and the observed peak (offset) and coefficient of regression $\mathrm{R}^{2}$ are recorded corresponding to different elements: $\mathrm{H}_{\delta}, \mathrm{H}_{\beta}, \mathrm{OIII}_{4960}, \mathrm{OIII}_{5007}$,
$\mathrm{NII}_{6584}, \mathrm{H}_{\alpha}, \mathrm{NII}_{6551}, \mathrm{SII}_{6717}$ and $\mathrm{SII}_{6733}$ responsible for the characteristic lines of the galaxy. We can see from Table 1 that, $\mathrm{OIII}_{5007}$ has a maximum intensity of $146.32 \times 10^{-17} \mathrm{erg} / \mathrm{s} / \mathrm{cm}^{2} / \AA$, corresponding to the central wavelength $5007 \AA$. The second strongest intensity is $116.84 \times 10^{-17}$ $\mathrm{erg} / \mathrm{s} / \mathrm{cm}^{2} / \AA$ corresponding to wavelength 6565 $\AA$, which is due to $\mathrm{H}_{\alpha}$ emission. The value of the coefficient of regression is more than $96 \%$ suggesting that the fitted curves almost agree with the Gaussian distribution. 
Table 1: Preferred Gaussian parameters of the Gaussian fits are listed in this Table.

\begin{tabular}{|l|l|l|l|l|l|l|l|l|l|}
\hline Elements & $\mathrm{H}_{\delta}$ & $\mathrm{H}_{\beta}$ & $\mathrm{OIII}_{4960}$ & $\mathrm{OIII}_{5007}$ & $\mathrm{NII}_{6584}$ & $\mathrm{H}_{\alpha}$ & $\mathrm{NII}_{6551}$ & SII $_{6717}$ & SII $_{6733}$ \\
\hline Wavelength & 4341 & 4862 & 4960 & 5007 & 6584 & 6565 & 6551 & 6717 & 6733 \\
\hline Intensity & 23.91 & 56.13 & 47.29 & 146.32 & 12.93 & 116.84 & 4.18 & 22.43 & 15.17 \\
\hline Area & 68.18 & 163.25 & 153.58 & 457.05 & 62.57 & 501.81 & 19.28 & 101.20 & 68.00 \\
\hline Height & 25.72 & 60.32 & 52.65 & 158.58 & 14.11 & 121.10 & 4.69 & 23.49 & 16.82 \\
\hline Offset & +0.14 & -0.29 & +0.44 & -0.43 & -0.70 & +0.36 & +1.32 & -0.47 & +0.66 \\
\hline $\mathbf{R}^{2}$ & 0.97 & 0.99 & 0.99 & 9.99 & 0.96 & 0.99 & 0.96 & 0.99 & 0.97 \\
\hline
\end{tabular}

The negative value of the offset of the Gaussian distribution of $\mathrm{H}_{\beta}, \mathrm{OIII}_{5007}, \mathrm{NII}_{6584}$, and $\mathrm{SII}_{6717}$ shows that the curves have negative skewness and they have a long left-hand tail as compared to righthand tail. In the same way, the positive value of the offset of the Gaussian distribution of $\mathrm{H}_{\delta}$, $\mathrm{OIII}_{4960}$, $\mathrm{H}_{\alpha}, \mathrm{NII}_{6551}$, and $\mathrm{SII}_{6733}$ shows that the curves have positive skewness and they have a long right-hand tail as compared to left-hand tail.

\section{Star Formation Rate}

Star Formation Rate (SFR) of the galaxy PGC 030133 calculated by using equation (2) is found to be $0.0029 \mathrm{M}_{\odot}$ year $^{-1}$ before extinction correction and $0.0033 \mathrm{M}_{\odot}$ year $^{-1}$ after extinction correction. We can see that the SFR of the galaxy calculated after extinction correction is more than that before correction indicating that the observed flux of the galaxy is more than the actual flux. The calculated value of SFR of the galaxy is remarkably low $\left(0.0033 \mathrm{M}_{\odot}\right.$ year $\left.^{-1}\right)$ as compared to its catalog value derived by using FUV flux. This is because of the large area coverage of FUV as compared to the SDSS 3" central region. It indicates that just a fraction of star formation occurs in the central part of the galaxy.

The line ratio $(c)=3.07$. This shows that the line ratio of the galaxies is slightly greater than its theoretical value (2.8). The higher value of $\mathrm{c}$ suggests the presence of dust at the center and the dust absorbs lower wavelength light more efficiently than higher wavelength light. The line ratio $\mathrm{NII}_{6584} / \mathrm{H}_{\alpha}=62.57 / 501.81=0.125$. Line metallicity calculated by using this line ratio from equation (3) is $12+\log (\mathrm{O} / \mathrm{H})=8.13$ dex which suggests that the PGC 030133 overall has metal content nearly three times lower than the sun.

\subsection{Photometry}

From the photometric calculations, several structural parameters of the galaxy PGC 030133 are obtained which are listed in Table 2. The first, second, third, fourth, fifth, and sixth columns represent the band of filter, mean surface brightness, effective radius, Sersic index, magnitude, and half-light radius respectively of $g$ and $\mathrm{z}$ bands.

Table 2: Global structural parameters of PGC 030133.

\begin{tabular}{|c|c|c|c|c|c|}
\hline Band & M. S. B.Mag/arc sec ${ }^{2}$ & $\mathbf{R}_{e}(\operatorname{arcsec})$ & $\mathbf{n}$ & mg (mag.) & $\mathbf{h} r(\operatorname{arcsec})$ \\
\hline $\mathrm{g}$ & 21.89 & 9.05 & 0.98 & 15.55 & 3.38 \\
\hline $\mathrm{z}$ & 21.13 & 8.00 & 0.89 & 14.92 & 3.05 \\
\hline
\end{tabular}

The angle made by the semi-major axis of the galaxy with the north celestial pole is called position angle and it gives the orientation of the galaxy. The radial profiles of position angle (PA) and ellipticity are shown in Fig. 3. From the figure, it seems that the position angle and ellipticity vary randomly as free parameters around the central region and it is almost constant in outer regions from the center of the galaxy both in g-band [Fig. 3(a)] and z-band [Fig. 3(b)].
From the Sersic modeling of the observed light profile in the parametric method, the effective radius of the galaxy is obtained to be 21.89 arcsec and $21.13 \mathrm{arcsec}$ in $\mathrm{g}$ and $\mathrm{z}$-bands respectively. The modeled major axis light profiles at g-band [Fig. 4(a)] and z-band [Fig. (b)] are shown in Fig. 4. It is found that the modeled best-fitted line perfectly coincides with the observed data and hence, the observed light profile can be well modeled with a simple Sersic function with a Sersic index of $\sim 0.9$ (near exponential). 
The half-life radius (hr) is obtained from the nonparametric approach that better represents the overall size of the galaxy. At first, a Petrosian radius $a_{p}$ is calculated at $\mathrm{n}=0.2$; as shown in Fig. 5(a) for g-band. It is assumed that radius $2 \mathrm{a}_{p}$ covers most of the galactic light and calculates a half-light radius for that total light as shown in Fig. 5(b). The same is done for

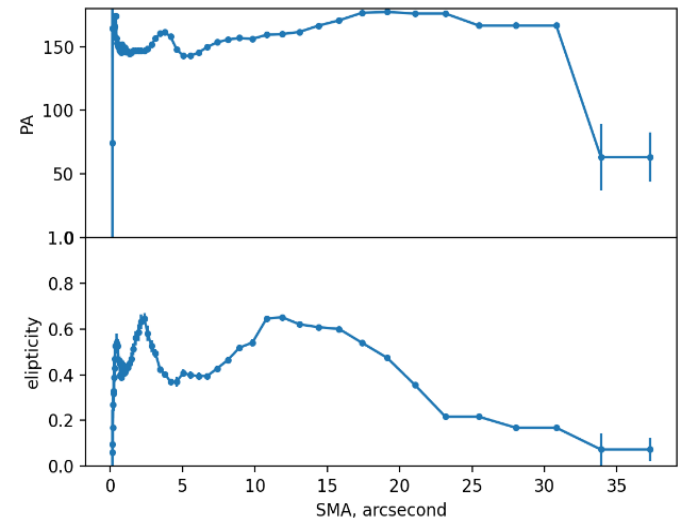

(a) g-band. the z-band as shown in Fig. 5. The derived Petrosian radius for this galaxy is 17.34 arcsec in g-band and 16.14 arcsec in z-band. Using the Petrosian method in the parametric approach, the half-light radii obtained are 3.38 arcsec and 3.05 arcsec respectively in $g$ and z-bands. Thus, we do not need to know the endpoint of the galaxy to find its size.

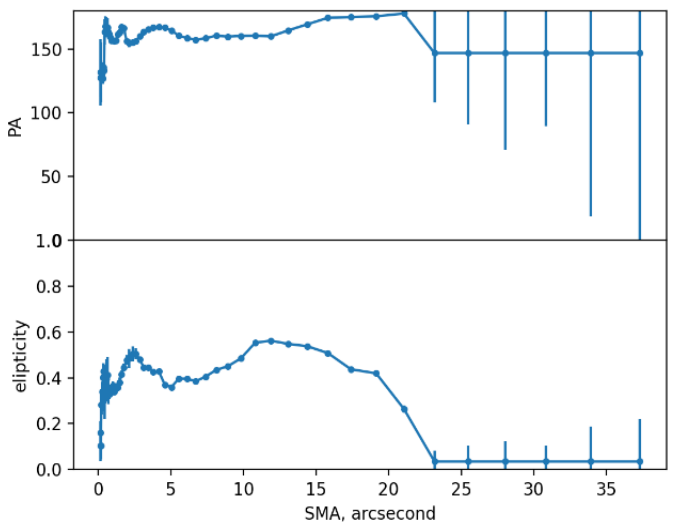

(b) z-band.

Fig. 3: Radial profiles of position angle (PA) and ellipticity

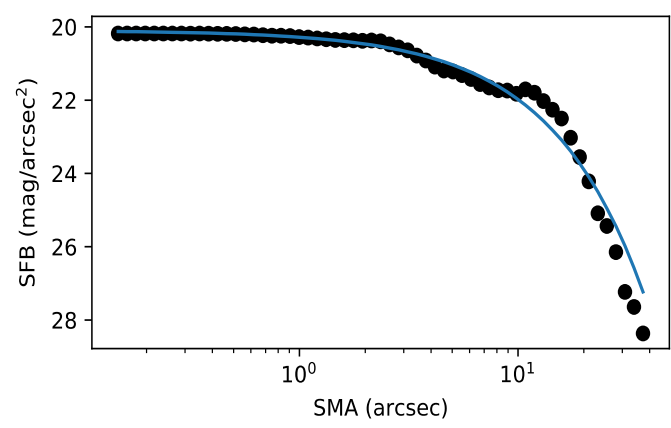

(a) g-band.

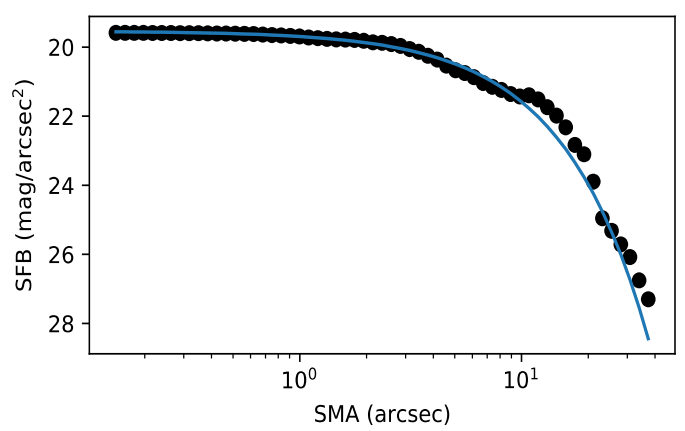

(b) $z$-band.

Fig. 4: Sersic modeling of the observed light profile of the galaxy PGC 030133

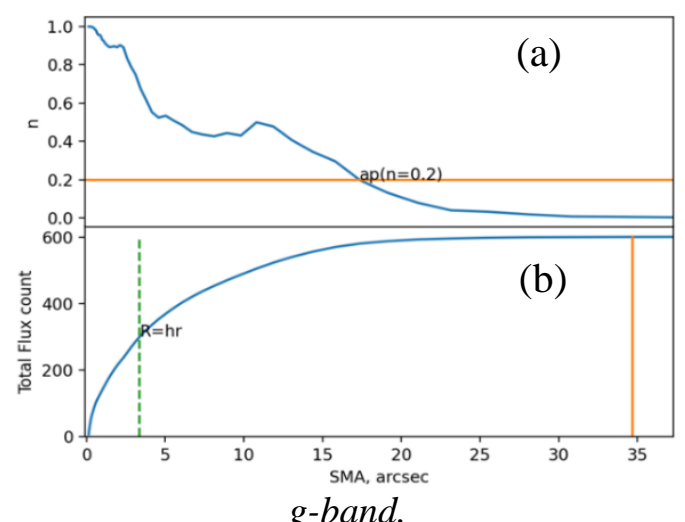

g-band.

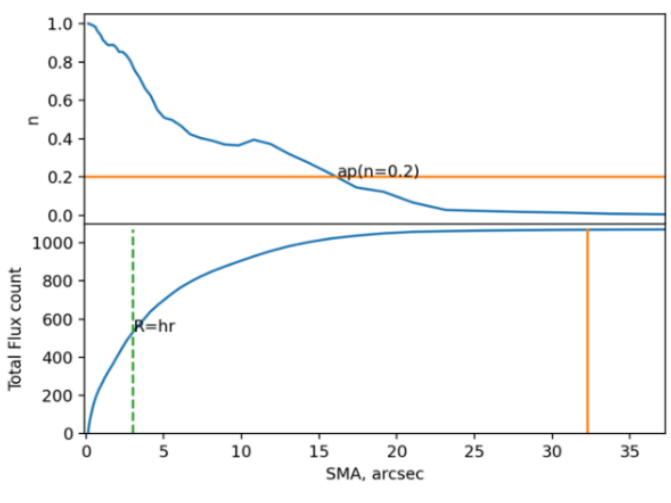

$z$-band.

Fig. 5: Radial profile of Petrosian index and cumulative intensity at g-band and z-band respectively. 


\section{Comparison with previous work}

We studied spectroscopic and morphological properties of a merging dwarf galaxy PGC 030133. It is a blue compact dwarf galaxy (BCD). Merging features in BCD-type galaxies are rare. Recent literature shows VCC 848 and UM 448 are the two well-studied BCD galaxies. An optical color of both VCC 848 and UM 448 is shown in Fig. 6. Zhang et al. have identified a shell feature BCD (VCC 848) in the Virgo cluster [24, 25]. Compare to VCC 848, PGC 030133 is one magnitude fainter and 0.2 dex bluer in g-r color. The color difference can be well explained by comparing their surrounding environment and gas mass fraction. VCC 848 has 5 times lower gas-mass fraction compared to PGC 030133. Both, VCC 848 and PGC 030133 have a similar half-light radius of $\sim 9$ ”. Another study of interacting BCD type galaxy is UM 448, located in a field environment [26]. A resolved spectroscopic study revealed that UM 448 is producing new stars at a staggering pace, i.e., 0.8 $\mathrm{M}_{\odot}$ year $^{-1}$, and has overall emission line metallicity $12+\log (\mathrm{O} / \mathrm{H})=8.15$ dex.
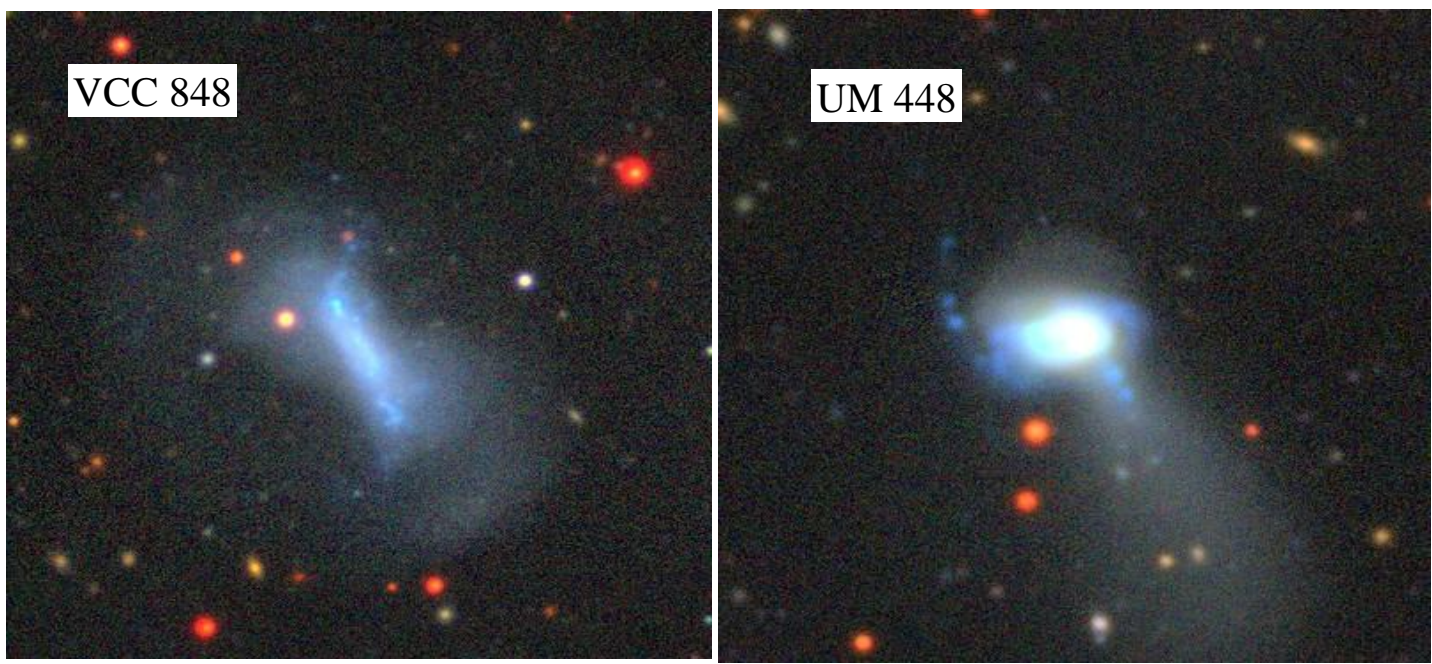

Fig. 6: Example of previously studied merging dwarf galaxies: VCC 848 and UM 448.

The images are obtained from the SDSS sky server.

\section{CONCLUSION}

From the spectroscopic and photometric analysis of the compact dwarf galaxy, PGC 030133 which is formed just after the merger, different conclusions are drawn based on the observations and calculations. The selected emission lines from the optical spectrum of galaxy PGC 030133 have a coefficient of regression, of more than $96 \%$ which indicates almost perfect agreement with Gaussian fits. The star formation rate and line metallicity are calculated by using $\mathrm{H}_{\alpha}$ flux. The derived SFR after extinction correction is $0.0033 \mathrm{M}_{\odot}$ year $^{-1}$ and its metallicity is $8.13 \mathrm{dex}$. This shows that the galaxy is a normal star-forming galaxy with total metal content three times lower than the Sun. A detailed morphological study of a compact dwarf galaxy, PGC 030133 is performed by using photometry and found that the major axis light profile can be well modeled with a simple Sersic function with near exponential Sersic index. The effective radius is calculated by modeling the observed light profile in the Sersic function and the half-light radius is calculated by the Petrosian method. The derived values of half-light radius are 3.38 arcsec and 3.05 arcsec in g-band and z-band respectively.

\section{ACKNOWLEDGEMENTS}

We acknowledge the University Grants Commission of Nepal, for financial support by providing a Ph.D. Fellowship and Research Support Grant (Award No.: PhD-75/76-S \& T-13) to carry out this research. We also acknowledge our anonymous referees for their valuable comments and suggestion. We would like to thank Mr. Rajesh Kumar Bachchan equally, for his remarkable efforts to help us in technical aspects.

This study is based on the archival images and spectra from the Sloan Digital Sky Survey (http://www:sdss.org/collaboration/credits.html).

\section{REFERENCES}

[1] Lotz, J. M.; Jonsson, P.; Cox, T. J.; Croton, D.; Primack, J. R.; Somerville, R. S. \& Stewart, K. The 
major and minor galaxy merger rates at $\mathrm{z}<1.5$. The Astrophysical Journal, 742(2): 103 (2011).

[2] Xu, C. \& Sulentic, J. W. Infrared emission in paired galaxies. II-Luminosity functions and farinfrared properties. The Astrophysical Journal, 374: 407-430 (1991).

[3] Sanders, D. B. \& Mirabel, I. F. Luminous infrared galaxies. Annual Review of Astronomy and Astrophysics, 34(1): 749-792 (1996).

[4] Dasyra, K. M.; Tacconi, L. J.; Davies, R. I.; Genzel, R.; Lutz, D.; Naab, T.; Burkert, A.; Veilleux, S. \& Sanders, D. B. Dynamical properties of ultraluminous infrared galaxies. I. Mass ratio conditions for ULIRG activity in interacting pairs. The Astrophysical Journal, 638(2): 745 (2006).

[5] Zwicky, F. Compact and Dispersed Cosmic Matter, Part II. Advances in Astronomy and Astrophysics, 7: 227-283 (1970). Elsevier.

[6] Zwicky, F. \& Zwicky, M. A. Catalogue of selected compact galaxies and post-eruptive galaxies: (1971). Guemligen: Zwicky.

[7] Hilker, M.; Infante, L.; Vieira, G.; Kissler-Patig, M. \& Richtler, T. The central region of the Fornax cluster-II. Spectroscopy and radial velocities of member and background galaxies. Astronomy and Astrophysics Supplement Series, 134(1): 75-86 (1999).

[8] Drinkwater, M. J.; Gregg, M. D.; Hilker, M.; Bekki, K.; Couch, W. J.; Ferguson, H. C. et al. A class of compact dwarf galaxies from disruptive processes in galaxy clusters. Nature, 423(6939): 519-521 (2003).

[9] Gil de Paz, A.; Madore, B. F. \& Pevunova, O. Palomar/Las Campanas Imaging Atlas of blue compact dwarf galaxies. I. Images and integrated photometry. Astrophysical journal supplement series, 147(1): 29-59 (2003).

[10] Pustilnik, S. A.; Kniazev, A. Y.; Lipovetsky, V. A. \& Ugryumov, A. V. Environment status of blue compact galaxies and trigger of star formation. Astronomy \& Astrophysics, 373(1): 24-37 (2001).

[11] Bekki, K. Formation of blue compact dwarf galaxies from merging and interacting gas-rich dwarfs. Monthly Notices of the Royal Astronomical Society: Letters, 388(1): L10-L14 (2008).

[12] Johnson, M.; Hunter, D. A.; Oh, S. H.; Zhang, H. X.; Elmegreen, B.; Brinks, E. et al. The stellar and gas kinematics of the LITTLE THINGS dwarf irregular galaxy NGC 1569. The Astronomical Journal, 144(5): 152 (2012).

[13] Ashley, T.; Elmegreen, B. G.; Johnson, M.; Nidever, D. L.; Simpson, C. E. \& Pokhrel, N. R. The HI chronicles of little things BCDs II: The origin of IC 10's HI structure. The Astronomical Journal, 148(6): 130 (2014).

[14] Paudel S.; Smith R.; Yoon S. J.; CalderónCastillo P. \& Duc P. A. A Catalog of Merging Dwarf Galaxies in the Local Universe. The Astrophysical Journal Supplement Series, 237(2): 1-20 (2018).

[15] Squires G. L. \& Squires, G. L. Practical physics. Cambridge University Press: (2001).

[16] Kennicutt Jr, R. C. Star formation in galaxies along the Hubble sequence. Annual Review of Astronomy and Astrophysics, 36(1): 189-231 (1998).

[17] Marino, R. A.; Rosales-Ortega, F. F.; Sánchez, S. F.; De Paz, A. G.; Vílchez, J.; Miralles-Caballero, D. et al. The $\mathrm{O} 3 \mathrm{~N} 2$ and $\mathrm{N} 2$ abundance indicators revisited: improved calibrations based on CALIFA and Te-based literature data. Astronomy \& Astrophysics, 559: A114 (2013).

[18] Osterbrock, D. E. Active galactic nuclei, Annals of the New York Academy of Science, 571: 99109 (1989).

[19] Calzetti, D.: Armus, L.; Bohlin, R. C.; Kinney, A. L.; Koornneef, J. \& Storchi-Bergmann, T., The Dust Content and Opacity of Actively Starforming Galaxies, The Astrophysical Journal, 533(2): 682-695 (2000).

[20] Jedrzejewski, R. I. CCD surface photometry of elliptical galaxies-I. Observations, reduction, and results. Monthly Notices of the Royal Astronomical Society, 226(4): 747-768 (1987).

[21] Sersic, J. L. Atlas de galaxias australes: (1968).

[22] Blanton, M. R.; Dalcanton, J.; Eisenstein, D.; Loveday, J.; Strauss, M. A.; SubbaRao, M. et al. The Luminosity function of galaxies in SDSS commissioning data. The Astronomical Journal, 121(5): 2358-2380 (2001).

[23] Petrosian, V. Surface brightness and evolution of galaxies. The Astrophysical Journal, 209: L1-L5 (1976).

[24] Zhang, H. X.; Smith, R.; Oh, S. H.; Paudel, S.; Duc, P. A.; Boselli, A. et al. The Blue Compact Dwarf Galaxy VCC 848 Formed by DwarfDwarf Merging: H I Gas, Star Formation, and Numerical Simulations. The Astrophysical Journal, 900(2): 152 (2020).

[25] Zhang, H. X.; Paudel, S.; Smith, R.; Duc, P. A.; Puzia, T. H.; Peng, E. W. et al. The Blue Compact Dwarf Galaxy VCC 848 Formed by Dwarf-Dwarf Merging. The Astrophysical Journal Letters, 891(1): L23 (2020).

[26] James, B. L.; Tsamis, Y. G.; Barlow, M. J.; Walsh, J. R. \& Westmoquette, M. S. The merging dwarf galaxy UM 448: chemodynamics of the ionized gas from VLT integral field spectroscopy. Monthly Notices of the Royal Astronomical Society, 428(1): 86-102 (2013). 